\title{
Temperley-Lieb Algebra: From Knot Theory to Logic and Computation via Quantum Mechanics
}

\section{Introduction}

Our aim in this paper is to trace some of the surprising and beautiful connections which are beginning to emerge between a number of apparently disparate topics.

\subsection{Knot Theory}

Vaughan Jones' discovery of his new polynomial invariant of knots in 1984 [26] triggered a spate of mathematical developments relating knot theory, topological quantum field theory, and statistical physics inter alia $[44,30]$. A central rôle, both in the initial work by Jones and in the subsequent developments, was played by what has come to be known as the Temperley-Lieb algebra. ${ }^{1}$

\subsection{Categorical Quantum Mechanics}

Recently, motivated by the needs of Quantum Information and Computation, Abramsky and Coecke have recast the foundations of Quantum Mechanics itself, in the more abstract language of category theory. The

${ }^{1}$ The original work of Temperley and Lieb [43] was in discrete lattice models of statistical physics. In finding exact solutions for a certain class of systems, they had identified the same relations which Jones, quite independently, found later in his work. 
key contribution is the paper [4], which develops an axiomatic presentation of quantum mechanics in the general setting of strongly compact closed categories, which is adequate for the needs of Quantum Information and Computation. Moreover, this categorical axiomatics can be presented in terms of a diagrammatic calculus which is both intuitive and effective, and can replace low-level computation with matrices by much more conceptual reasoning. This diagrammatic calculus can be seen as a proof system for a logic [6], leading to a radically new perspective on what the right logical formulation for Quantum Mechanics should be.

This line of work has a direct connection to the Temperley-Lieb algebra, which can be put in a categorical framework, in which it can be described essentially as the free pivotal dagger category on one self-dual generator [21]. ${ }^{2}$ Here pivotal dagger category is a non-symmetric ("planar") version of (strongly or dagger) compact closed category — the key notion in the Abramsky-Coecke axiomatics.

\subsection{Logic and Computation}

The Temperley-Lieb algebra itself has some direct and striking connections to basic ideas in Logic and Computation, which offer an intriguing and promising bridge between these prima facie very different areas. We shall focus in particular on the following two topics:

- The Temperley-Lieb algebra has always hitherto been presented as a quotient of some sort: either algebraically by generators and relations as in Jones' original presentation [26], or as a diagram algebra modulo planar isotopy as in Kauffman's presentation [29]. We shall use tools from Geometry of Interaction [23], a dynamical interpretation of proofs under Cut Elimination developed as an off-shoot of Linear Logic [22], to give a direct description of the Temperley-Lieb category - a fully abstract presentation, in Computer Science terminology [37]. This also brings something new to the Geometry of Interaction, since we are led to develop a planar version of it, and to verify that the interpretation of CutElimination (the "Execution Formula" [23], or "composition by feedback" $[8,1])$ preserves planarity.

${ }^{2}$ Strictly speaking, the full Temperley-Lieb category over a ring $R$ is the free $R$-linear enrichment of this free pivotal dagger category. 
- We shall also show how the Temperley-Lieb algebra provides a natural setting in which computation can be performed diagrammatically as geometric simplification - "yanking lines straight". We shall introduce a "planar $\lambda$-calculus" for this purpose, and show how it can be interpreted in the Temperley-Lieb category.

\subsection{Outline of the Paper}

We briefly summarize the further contents of this paper. In Section 2 we introduce the Temperley-Lieb algebras, emphasizing Kauffman's diagrammatic formulation. We also briefly outline how the Temperley-Lieb algebra figures in the construction of the Jones polynomial. In Section 3 we describe the Temperley-Lieb category, which provides a more structured perspective on the Temperley-Lieb algebras. In Section 4, we discuss some features of this category, which have apparently not been considered previously, namely a characterization of monics and epics, leading to results on image factorization and splitting of idempotents. In Section 5, we briefly discuss the connections with the Abramsky-Coecke categorical formulation of Quantum Mechanics, and raise some issues and questions about the possible relationship betwen planar, braided and symmetric settings for Quantum Information and Computation. In Section 6 we develop a planar version of Geometry of Interaction, and the direct "fully abstract" presentation of the Temperley-Lieb category. In Section 7 we discuss the planar $\lambda$-calculus and its interpretation in the Temperley-Lieb category. We conclude in Section 8 with some further directions.

Note to the Reader Since this paper aims at indicating crosscurrents between several fields, it has been written in a somewhat expansive style, and an attempt has been made to explain the context of the various ideas we will discuss. We hope it will be accessible to readers with a variety of backgrounds.

\section{The Temperley-Lieb Algebra}

Our starting point is the Temperley-Lieb algebra, which has played a central role in the discovery by Vaughan Jones of his new polynomial invariant of knots and links [26], and in the subsequent developments 
over the past two decades relating knot theory, topological quantum field theory, and statistical physics [30].

Jones' approach was algebraic: in his work, the Temperley-Lieb algebra was originally presented, rather forbiddingly, in terms of abstract generators and relations. It was recast in beautifully elementary and conceptual terms by Louis Kauffman as a planar diagram algebra [29]. We begin with the algebraic presentation.

\subsection{Temperley-Lieb algebra: generators and relations}

We fix a ring $R$; in applications to knot polynomials, this is taken to be a ring of Laurent polynomials $\mathbb{C}\left[X, X^{-1}\right]$. Given a choice of parameter $\tau \in R$ and a dimension $n \in \mathbb{N}$, we define the Temperley-Lieb algebra $\mathcal{A}_{n}(\tau)$ to be the unital, associative $R$-linear algebra with generators

$$
U_{1}, \ldots, U_{n-1}
$$

and relations

$$
\begin{aligned}
U_{i} U_{j} U_{i} & =U_{i} & & |i-j|=1 \\
U_{i}^{2} & =\tau \cdot U_{i} & & \\
U_{i} U_{j} & =U_{j} U_{i} & & |i-j|>1
\end{aligned}
$$

Note that the only relations used in defining the algebra are multiplicative ones. This suggests that we can obtain the algebra $\mathcal{A}_{n}(\tau)$ by presenting the multiplicative monoid $\mathcal{M}_{n}$, and then obtaining $\mathcal{A}_{n}(\tau)$ as the monoid algebra of formal $R$-linear combinations $\sum_{i} r_{i} \cdot a_{i}$ over $\mathcal{M}_{n}$, with the multiplication in $\mathcal{A}_{n}(\tau)$ defined as the bilinear extension of the monoid multiplication in $\mathcal{M}_{n}$ :

$$
\left(\sum_{i} r_{i} \cdot a_{i}\right)\left(\sum_{j} s_{j} \cdot b_{j}\right)=\sum_{i, j}\left(r_{i} s_{j}\right) \cdot\left(a_{i} b_{j}\right) .
$$

We define $\mathcal{M}_{n}$ as the monoid with generators

$$
\delta, U_{1}, \ldots, U_{n-1}
$$

and relations

$$
\begin{array}{rlrl}
U_{i} U_{j} U_{i} & =U_{i} & & |i-j|=1 \\
U_{i}^{2} & =\delta U_{i} & \\
U_{i} U_{j} & =U_{j} U_{i} & & |i-j|>1 \\
\delta U_{i} & =U_{i} \delta &
\end{array}
$$


We can then obtain $\mathcal{A}_{n}(\tau)$ as the monoid algebra over $\mathcal{M}_{n}$, subject to the identification

$$
\delta=\tau \cdot 1
$$

\subsection{Diagram Monoids}

These formal algebraic ideas are brought to vivid geometric life by Kauffman's interpretation of the monoids $\mathcal{M}_{n}$ as diagram monoids.

We start with two parallel rows of $n$ dots (geometrically, the dots are points in the plane). The general form of an element of the monoid is obtained by "joining up the dots" pairwise in a smooth, planar fashion, where the arc connecting each pair of dots must lie within the rectangle framing the two parallel rows of dots. Such diagrams are identified up to planar isotopy, i.e. continuous deformation within the portion of the plane bounded by the framing rectangle.

Thus the generators $U_{1}, \ldots, U_{n-1}$ can be drawn as follows:

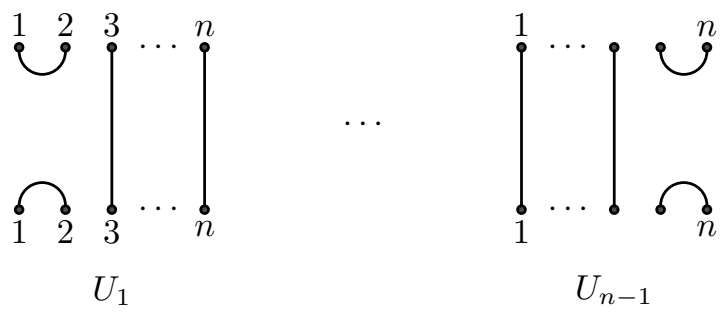

The generator $\delta$ corresponds to a loop $\bigcirc$ - all such loops are identified up to isotopy.

We refer to arcs connecting dots in the top row as cups, those connecting dots in the bottom row as caps, and those connecting a dot in the top row to a dot in the bottom row as through lines.

Multiplication $x y$ is defined by identifying the bottom row of $x$ with the top row of $y$, and composing paths. In general loops may be formed - these are "scalars", which can float freely across these figures. The relations can be illustrated as follows:

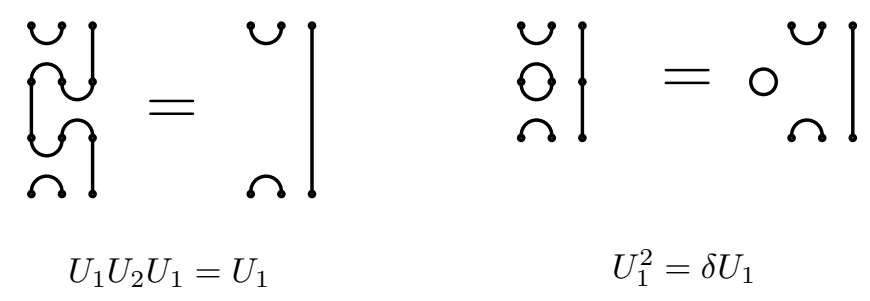




$$
\underset{\Omega}{\cup}=\underset{\Omega}{\bigcup_{U} U_{3}=U_{3} U_{1}}
$$

\subsection{Expressiveness of the Generators}

The fact that all planar diagrams can be expressed as products of generators is not entirely obvious. For proofs, see [29, 20]. As an illustrative example, consider the planar diagrams in $\mathcal{M}_{3}$. Apart from the generators $U_{1}, U_{2}$, and ignoring loops, there are three:
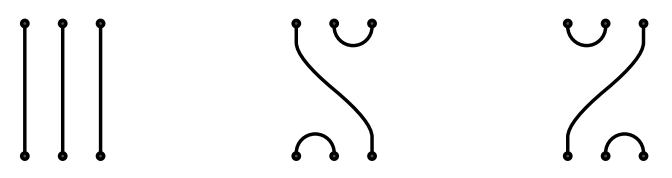

The first is the identity for the monoid; we refer to the other two as the left wave and right wave respectively. The left wave can be expressed as the product $U_{2} U_{1}$ :

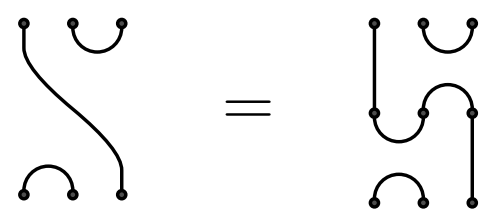

The right wave has a similar expression.

Once we are in dimension four or higher, we can have nested cups and caps. These can be built using waves, as illustrated by the following:

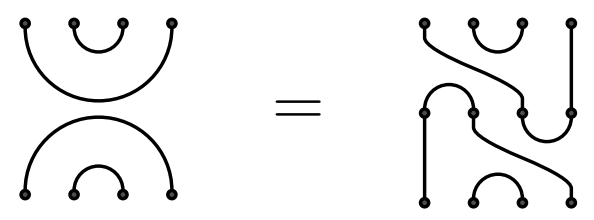

\subsection{The Trace}

There is a natural trace function on the Temperley-Lieb algebra, which can be defined diagrammatically on $\mathcal{M}_{n}$ by connecting each dot in the top row to the corresponding dot in the bottom row, using auxiliary cups and cups. This always yields a diagram isotopic to a number of 
loops - hence to a scalar, as expected. This trace can then be extended linearly to $\mathcal{A}_{n}(\tau)$.

We illustrate this firstly by taking the trace of a wave - which is equal to a single loop:

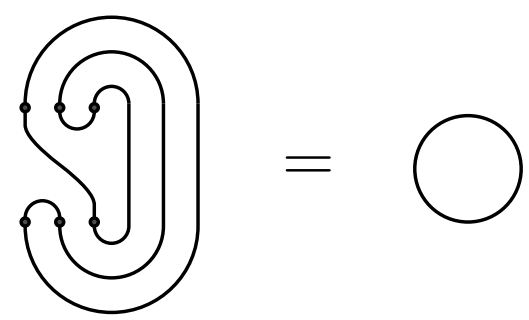

The Ear is a Circle

Our second example illustrates the important general point that the trace of the identity in $\mathcal{M}_{n}$ is $\delta^{n}$ :

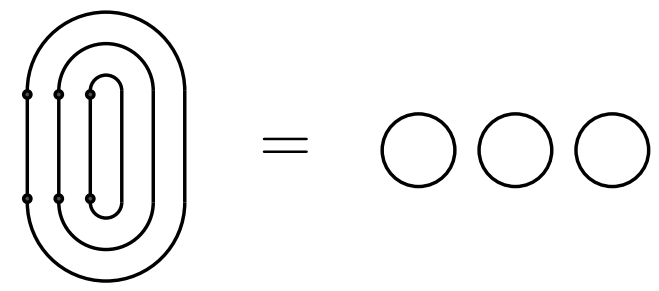

\subsection{The Connection to Knots}

How does this connect to knots? Again, a key conceptual insight is due to Kauffman, who saw how to recast the Jones polynomial in elementary combinatorial form in terms of his bracket polynomial. The basic idea of the bracket polynomial is expressed by the following equation:

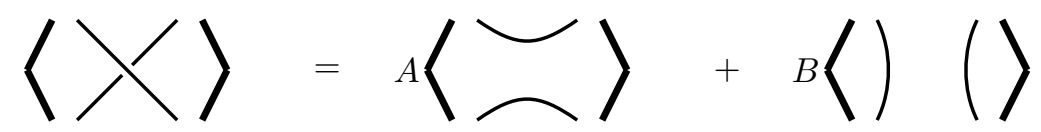

Each over-crossing in a knot or link is evaluated to a weighted sum of the two possible planar smoothings. With suitable choices for the coefficients $A$ and $B$ (as Laurent polynomials), this is invariant under the second and third Reidemeister moves. With an ingenious choice of normalizing factor, it becomes invariant under the first Reidemeister move - and yields the Jones polynomial! What this means algebraically is that the braid group $\mathcal{B}_{n}$ has a representation in the Temperley-Lieb 
algebra $\mathcal{A}_{n}(\tau)$ - the above bracket evaluation shows how the generators $\beta_{i}$ of the braid group are mapped into the Temperley-Lieb algebra:

$$
\beta_{i} \mapsto A \cdot U_{i}+B \cdot 1 .
$$

Every knot arises as the closure (i.e. the diagrammatic trace) of a braid; the invariant arises by mapping the open braid into the Temperley-Lieb algebra, and taking the trace there.

This is just the beginning of a huge swathe of further developments, including: Topological Quantum Field Theories [44], Quantum Groups [28], Quantum Statistical mechanics [30], Diagram Algebras and Representation Theory [25], and more.

\section{The Temperley-Lieb Category}

We can expose more structure by gathering all the Temperley-Lieb algebras into a single category. We begin with the category $\mathcal{D}$ which plays a similar role with respect to the diagram monoids $\mathcal{M}_{n}$.

The objects of $\mathcal{D}$ are the natural numbers. An arrow $\mathbf{n} \rightarrow \mathbf{m}$ is given by

- a number $k \in \mathbb{N}$ of loops

- a diagram which joins the top row of $n$ dots and the bottom row of $m$ dots up pairwise, in the same smooth planar fashion as we have already specified for the diagram monoids. As before, diagrams are identified up to planar isotopy.

Composition of arrows $f: \mathbf{n} \rightarrow \mathbf{m}$ and $g: \mathbf{m} \rightarrow \mathbf{p}$ is defined by identifying the bottom row of $m$ dots for $f$ with the top row of $m$ dots for $g$, and composing paths. The loops in the resulting arrow are those of $f$ and of $g$, together with any formed by the process of composing paths.

Clearly we recover each $\mathcal{M}_{n}$ as the endomorphism monoid $\mathcal{D}(\mathbf{n}, \mathbf{n})$. Moreover, we can define the Temperley-Lieb category $\mathcal{T}$ over a ring $R$ as the free $R$-linear category generated by $\mathcal{D}$, with a construction which generalizes that of the monoid algebra: the objects of $\mathcal{T}$ are the same as those of $\mathcal{D}$, and arrows are $R$-linear combinations of arrows of $\mathcal{D}$, with composition defined by bilinear extension from that in $\mathcal{D}$ :

$$
\left(\sum_{i} r_{i} \cdot g_{i}\right) \circ\left(\sum_{j} s_{j} \cdot f_{j}\right)=\sum_{i, j}\left(r_{i} s_{j}\right) \cdot\left(g_{i} \circ f_{j}\right) \text {. }
$$


If we fix a parameter $\tau \in R$, then we obtain the category $\mathcal{T}_{\tau}$ by the identification of the loop $\bigcirc$ in $\mathcal{D}$ with the scalar $\tau$ in $\mathcal{T}{ }^{3}$ We then recover the Temperley-Lieb algebras as

$$
\mathcal{A}_{n}(\tau)=\mathcal{T}_{\tau}(\mathbf{n}, \mathbf{n}) .
$$

New possibilities also arise in $\mathcal{D}$. In particular, we get the pure cap

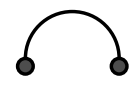

as (the unique) arrow $\mathbf{0} \rightarrow \mathbf{2}$, and similarly the pure cup as the unique arrow $\mathbf{2} \rightarrow \mathbf{0}$. More generally, for each $n$ we have arrows $\eta_{\mathbf{n}}: \mathbf{0} \rightarrow \mathbf{n}+\mathbf{n}$, and $\epsilon_{\mathbf{n}}: \mathbf{n}+\mathbf{n} \rightarrow \mathbf{0}$ :
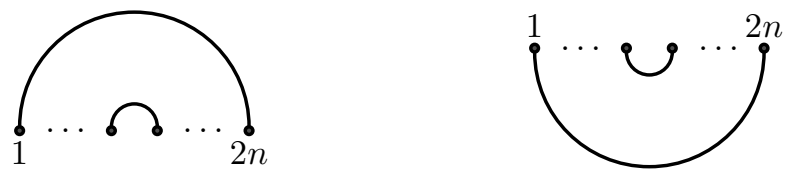

We refer to the arrows $\eta_{\mathbf{n}}$ as units, and the arrows $\epsilon_{\mathbf{n}}$ as counits.

The category $\mathcal{D}$ has a natural strict monoidal structure. On objects, we define $\mathbf{n} \otimes \mathbf{m}=\mathbf{n}+\mathbf{m}$, with unit given by $I=\mathbf{0}$. The tensor product of morphisms

$$
\frac{f: \mathbf{n} \rightarrow \mathbf{m} \quad g: \mathbf{p} \rightarrow \mathbf{q}}{f \otimes g: \mathbf{n}+\mathbf{p} \rightarrow \mathbf{p}+\mathbf{q}}
$$

is given by juxtaposition of diagrams in the evident fashion, with (multiset) union of loops. Thus we can write the units and counits as arrows

$$
\eta_{\mathbf{n}}: I \rightarrow \mathbf{n} \otimes \mathbf{n}, \quad \epsilon_{\mathbf{n}}: \mathbf{n} \otimes \mathbf{n} \rightarrow I .
$$

These units and counits satisfy important identities, which we illustrate diagrammatically

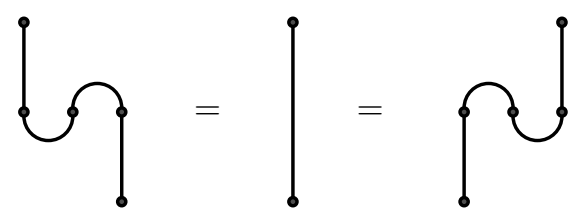

and write algebraically as

$$
\left(\epsilon_{\mathbf{n}} \otimes 1_{\mathbf{n}}\right) \circ\left(1_{\mathbf{n}} \otimes \eta_{\mathbf{n}}\right)=1_{\mathbf{n}}=\left(1_{\mathbf{n}} \otimes \epsilon_{\mathbf{n}}\right) \circ\left(\eta_{\mathbf{n}} \otimes 1_{\mathbf{n}}\right) .
$$

${ }^{3}$ The full justification of this step requires the identification of $\mathcal{D}$ as a free pivotal category, as discussed below. 


\subsection{Pivotal Categories}

From these observations, we see that $\mathcal{D}$ is a strict pivotal category [21], in which the duality on objects is trivial: $A=A^{*}$. We recall that a strict pivotal category is a strict monoidal category $(\mathcal{C}, \otimes, I)$ with an assignment $A \mapsto A^{*}$ on objects satisfying

$$
A^{* *}=A, \quad(A \otimes B)^{*}=B^{*} \otimes A^{*}, \quad I^{*}=I,
$$

and for each object $A$, arrows

$$
\eta_{A}: I \rightarrow A^{*} \otimes A, \quad \epsilon_{A}: A \otimes A^{*} \rightarrow I
$$

satisfying the triangular identities:

$$
\left(\epsilon_{A} \otimes 1_{A}\right) \circ\left(1_{A} \otimes \eta_{A}\right)=1_{A}, \quad\left(1_{A^{*}} \otimes \epsilon_{A}\right) \circ\left(\eta_{A} \otimes 1_{A^{*}}\right)=1_{A^{*}} .
$$

In addition, the following coherence equations are required to hold:

$$
\eta_{I}=1_{I}, \quad \eta_{A \otimes B}=\left(1_{B *} \otimes \eta_{A} \otimes 1_{B}\right) \circ \eta_{B},
$$

and, for $f: A \rightarrow B$ :

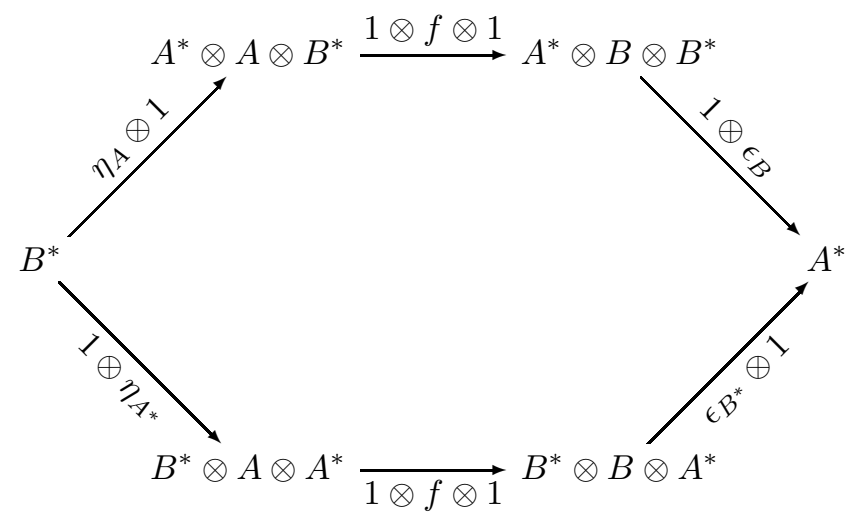

This last equation is illustrated diagrammatically by

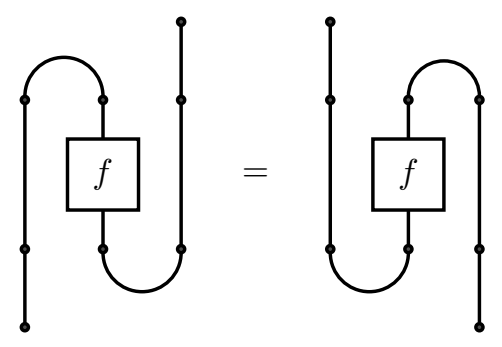


We extend ()* to a contravariant involutive functor:

$$
\frac{f: A \rightarrow B}{f^{*}: B^{*} \rightarrow A^{*}} \quad f^{*}=\left(1 \otimes \epsilon_{A}\right) \circ(1 \otimes f \otimes 1) \circ\left(\eta_{A} \otimes 1\right)
$$

which indeed satisfies

$$
1^{*}=1, \quad(g \circ f)^{*}=f^{*} \circ g^{*}, \quad f^{* *}=f,
$$

the last equation being illustrated diagrammatically by

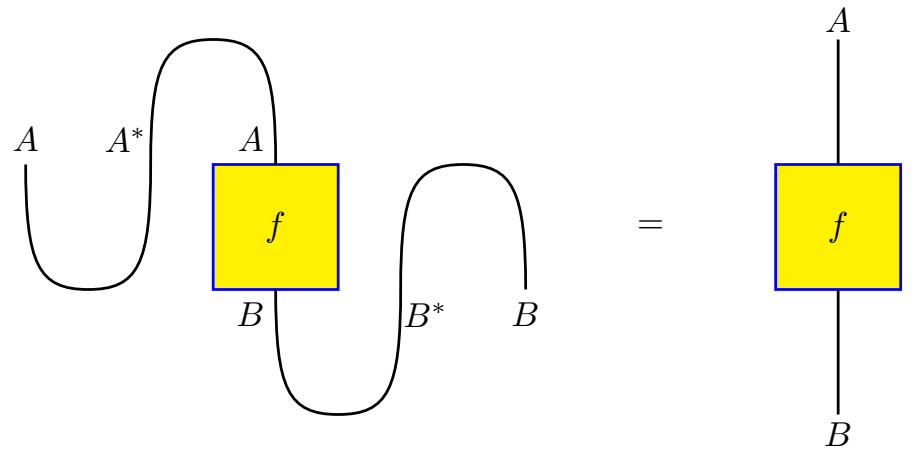

These axioms have powerful consequences. In particular, $\mathcal{C}$ is monoidal closed, with internal hom given by $A^{*} \otimes B$, and the adjunction:

$$
\mathcal{C}(A \otimes B, C) \simeq \mathcal{C}\left(B, A^{*} \otimes C\right):: f \mapsto(1 \otimes f) \circ\left(\eta_{A} \otimes 1\right) .
$$

This means that a restricted form of $\lambda$-calculus can interpreted in such categories - a point we shall return to in Section 7 .

A trace function can be defined in pivotal categories, which takes an endmorphism $f: A \rightarrow A$ to a scalar in $\mathcal{C}(I, I)$ :

$$
\operatorname{Tr}(f)=\epsilon_{A} \circ(f \otimes 1) \circ \eta_{A^{*}} .
$$

It satisfies:

$$
\operatorname{Tr}(g \circ f)=\operatorname{Tr}(f \circ g) .
$$

In $\mathcal{D}$, this definition yields exactly the diagrammatic trace we discussed previously.

We have the following important characterization of the diagrammatic category $\mathcal{D}$ : 


\section{PROPOSITION 1.1}

$\mathcal{D}$ is the free pivotal category over one self-dual generator; that is, freely generated over the one-object one-arrow category, with object $A$ say, subject to the equation $A=A^{*}$.

This was mentioned (although not proved) in [21]; see also [20]. The methods in [3] can be adapted to prove this result, using the ideas we shall develop in Section 6.

The idea of "identifying the loop with the scalar $\tau$ " in passing from $\mathcal{D}$ to the full Temperley-Lieb category $\mathcal{T}_{\tau}$ can be made precise using the construction given in [3] of gluing a specified ring $R$ of scalars onto a free compact closed category, along a given map from the loops in the generating category to $R$. In this case, there is a single loop in the generating category, and we send it to $\tau$.

\subsection{Pivotal Dagger Categories}

We now mention a strengthening of the axioms for pivotal categories, corresponding to the notion of strongly compact closed or dagger compact closed category which has proved to be important in the categorical approach to Quantum Mechanics [4, 5]. Again we give the strict version for simplicity. We assume that the strict monoidal category $(\mathcal{C}, \otimes, I)$ comes equipped with an identity-on-objects, contravariant involutive functor ()$^{\dagger}$ such that $\epsilon_{A}=\eta_{A^{*}}^{\dagger}$. The idea is that $f^{\dagger}$ abstracts from the adjoint of a linear map, and allows the extra structure arising from the use of complex Hilbert spaces in Quantum Mechanics to be expressed in the abstract setting.

Note that there is a clear diagrammatic distinction between the dual $f^{*}$ and the adjoint $f^{\dagger}$. The dual corresponds to $180^{\circ}$ rotation in the plane:

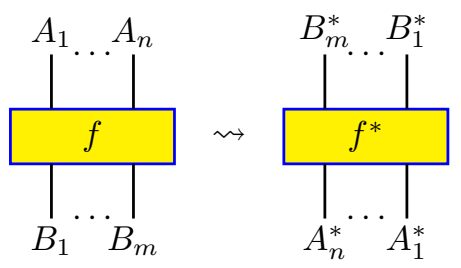

while the adjoint is reflection in the $x$-axis: 

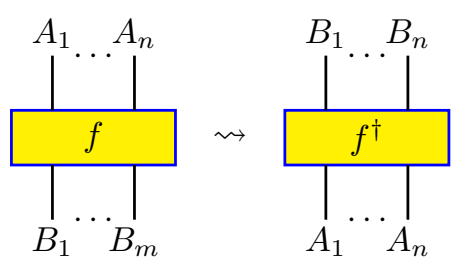

For example in $\mathcal{D}$, if we consider the left and right wave morphisms $L$ and $R$ :
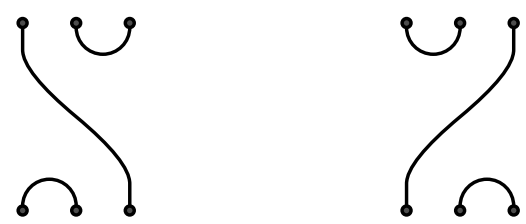

then we have

$$
L^{*}=L, \quad L^{\dagger}=R, \quad R^{*}=R, \quad R^{\dagger}=L .
$$

Using the adjoint, we can define a covariant functor

$$
\frac{f: A \rightarrow B}{f_{*}: A^{*} \rightarrow B^{*}} \quad f \mapsto f^{* \dagger}
$$

We have

$$
\left(f^{*}\right)_{*}=f^{\dagger}=\left(f_{*}\right)^{*} .
$$

In terms of complex matrices, $f^{*}$ is transpose, while $f_{*}$ is complex conjugation. Diagrammatically, $f_{*}$ is "reflection in the $y$-axis".

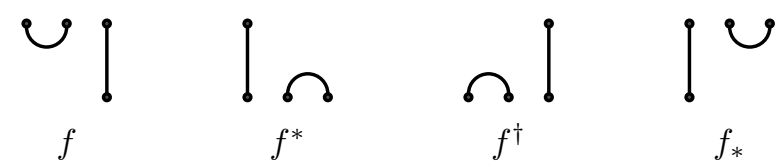

We have the following refinement of Proposition 1.1, by similar methods to those used for free strongly compact closed categories in [3].

PROPOSITION 1.2

$\mathcal{D}$ is the free pivotal dagger category over one self-dual generator. 


\section{Factorization and Idempotents}

We now consider some structural properties of the category $\mathcal{D}$ which we have not found elsewhere in the literature. ${ }^{4}$

We begin with a pleasingly simple diagrammatic characterization of monics and epics in $\mathcal{D}$.

\section{PROPOSITION 1.3}

An arrow in $\mathcal{D}$ is monic iff it has no cups; it is epic iff it has no caps.

Proof Suppose that $f: \mathbf{n} \rightarrow \mathbf{m}$ has no cups. Thus all dots in $\mathbf{n}$ are connected by through lines to dots in $\mathbf{m}$. Now consider a composition $f \circ g$. No loops can be formed by this composition; hence we can recover $g$ from $f \circ g$ by erasing the caps of $f$. Moreover, the number of loops in $f \circ g$ will simply be the sum of the loops in $f$ and $g$, so we can recover the loops of $g$ by subtracting the loops of $f$ from the composition. It follows that

$$
f \circ g=f \circ h \Longrightarrow g=h,
$$

i.e. that $f$ is monic, as required.

For the converse, suppose that $f$ has a cup, which we can assume to be connecting dots $i$ and $i+1$ in the top row. (Note that if $i<j$ are connected by a cup, then by planarity, every $k$ with $i<k<j$ must also be connected in a cup to some $l$ with $i<l<j$.) Then $f \circ \delta \cdot 1=f \circ\left(1 \otimes U_{i} \otimes 1\right)$, so $f$ is not monic. Diagrammatically, this says that we can either form a loop using the cup of $f$, or simply add a loop which is attached to an identity morphism.

The characterization of epics is entirely similar.

This immediately yields an "image factorization" structure for $\mathcal{D}$.

\section{PROPOSITION 1.4}

Every arrow in $\mathcal{D}$ has an epi-mono factorization.

Proof Given an arrow $f: \mathbf{n} \rightarrow \mathbf{m}$, suppose it has $p$ cups and $q$ caps. Then we obtain arrows $e: \mathbf{n} \rightarrow(\mathbf{m}-2 \mathbf{q})$ by erasing the caps, and $m:(\mathbf{n}-2 \mathbf{p}) \rightarrow \mathbf{m}$ by erasing the cups. By Proposition $1.3, e$ is epic and

${ }^{4}$ The idea of considering these properties arose from a discussion with Louis Kauffman, who showed the author a direct diagrammatic characterization of idempotents in $\mathcal{D}$, which has subsequently appeared in [32]. 
$m$ monic. Moreover, the number of dots in the top and bottom rows connected by through lines must be the same. Hence

$$
(\mathbf{m}-2 \mathbf{q})=\mathbf{k}=(\mathbf{n}-2 \mathbf{p}),
$$

and we can compose $e$ and $m$ to recover $f$. Note that by planarity, once we have assigned cups and caps, there is no choice about the correspondence between top and bottom row dots by through lines.

This factorization is "essentially" unique. However, we are free to split the $l$ loops of $f$ between $e$ and $m$ in any way we wish, so there is a distinct factorization $\delta^{a} \cdot m \circ \delta^{b} \cdot e$ for all $a, b \in \mathbb{N}$ with $a+b=l$.

We illustrate the epi-mono factorization for the left wave:

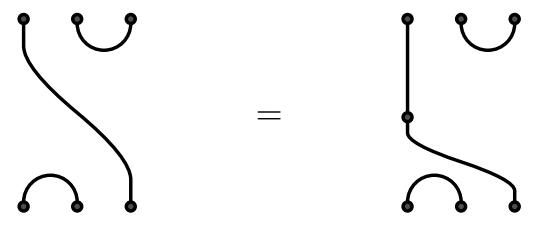

We recall that an idempotent in a category is an arrow $i: A \rightarrow A$ such that $i^{2}=i$. We say that an idempotent $i$ splits if there are arrows $r: A \rightarrow B$ and $s: B \rightarrow A$ such that

$$
i=s \circ r, \quad r \circ s=1_{B} .
$$

\section{PROPOSITION 1.5}

All idempotents split in $\mathcal{D}$.

Proof Let $i: \mathbf{n} \rightarrow \mathbf{n}$ be an idempotent in $\mathcal{D}$. By Proposition 1.4, $i=m \circ e$, where $e: \mathbf{n} \rightarrow \mathbf{k}$ is epic and $m: \mathbf{k} \rightarrow \mathbf{n}$ is monic. Now

$$
m \circ e \circ m \circ e=m \circ e .
$$

Since $m$ is monic, this implies that $e \circ m \circ e=e=1 \circ e$. Since $e$ is epic, this implies that $e \circ m=1$.

\section{Categorical Quantum Mechanics}

We now relate our discussion to the Abramsky-Coecke programme of Categorical Quantum Mechanics. 
This approach is very different to previous work on the Computer Science side of this interdisciplinary area, which has focussed on quantum algorithms and complexity. The focus has rather been on developing high-level methods for Quantum Information and Computation (QIC)languages, logics, calculi, type systems etc.-analogous to those which have proved so essential in classical computing [2]. This has led to nothing less than a recasting of the foundations of Quantum Mechanics itself, in the more abstract language of category theory. The key contribution is the paper with Coecke [4], in which we develop an axiomatic presentation of quantum mechanics in the general setting of strongly compact closed categories, which is adequate for all the needs of QIC.

Specifically, we show that we can recover the key quantum mechanical notions of inner-product, unitarity, full and partial trace, HilbertSchmidt inner-product and map-state duality, projection, positivity, measurement, and Born rule (which provides the quantum probabilities), axiomatically at this high level of abstraction and generality. Moreover, we can derive the correctness of protocols such as quantum teleportation, entanglement swapping and logic-gate teleportation $[10,24,45]$ in a transparent and very conceptual fashion. Also, while at this level of abstraction there is no underlying field of complex numbers, there $i s$ still an intrinsic notion of 'scalar', and we can still make sense of dual vs. adjoint $[4,5]$, and global phase and elimination thereof [15]. Peter Selinger recovered mixed state, complete positivity and Jamiolkowski map-state duality [42]. Recently, in collaboration with Dusko Pavlovic and Eric Paquette, decoherence, generalized measurements and Naimark's theorem have been recovered $[17,16]$.

Moreover, this formalism has two important additional features. Firstly, it goes beyond the standard Hilbert-space formalism, in that it is able to capture classical as well as quantum information flows, and the interaction between them, within the formalism. For example, we can capture the idea that the result of a measurement is used to determine a further stage of quantum evolution, as e.g. in the teleportation protocol [10], where a unitary correction must be performed after a measurement; or also in measurement-based quantum computation [39, 40]. Secondly, this categorical axiomatics can be presented in terms of a diagrammatic calculus which is extremely intuitive, and potentially can replace lowlevel computation with matrices by much more conceptual - and automatable - reasoning. Moreover, this diagrammatic calculus can be seen as a proof system for a logic, leading to a radically new perspective on what the right logical formulation for Quantum Mechanics should 
be. This latter topic is initiated in [6], and developed further in the forthcoming thesis of Ross Duncan.

\subsection{Outline of the approach}

We now give some further details of the approach. The general setting is that of strongly (or dagger) compact closed categories, which are the symmetric version of the pivotal dagger categories we encountered in Section 3. Thus, in addition to the structure mentioned there, we have a symmetry natural isomorphism

$$
\sigma_{A, B}: A \otimes B \simeq B \otimes A .
$$

See [5] for an extended discussion. An important feature of the AbramskyCoecke approach is the use of an intuitive graphical calculus, which is essentially the diagrammatic formalism we have seen in the TemperleyLieb setting, extended with more general basic types and arrows. The key point is that this formalism admits a very direct physical interpretation in Quantum Mechanics.

In the graphical calculus we depict physical processes by boxes, and we label the inputs and outputs of these boxes by types which indicate the kind of system on which these boxes act, e.g. one qubit, several qubits, classical data, etc. Sequential composition (in time) is depicted by connecting matching outputs and inputs by wires, and parallel composition (tensor) by locating entities side by side e.g.

$1_{A}: A \rightarrow A \quad f: A \rightarrow B \quad g \circ f \quad 1_{A} \otimes 1_{B} \quad f \otimes 1_{C} \quad f \otimes g \quad(f \otimes g) \circ h$

for $g: B \rightarrow C$ and $h: E \rightarrow A \otimes B$ are respectively depicted as:

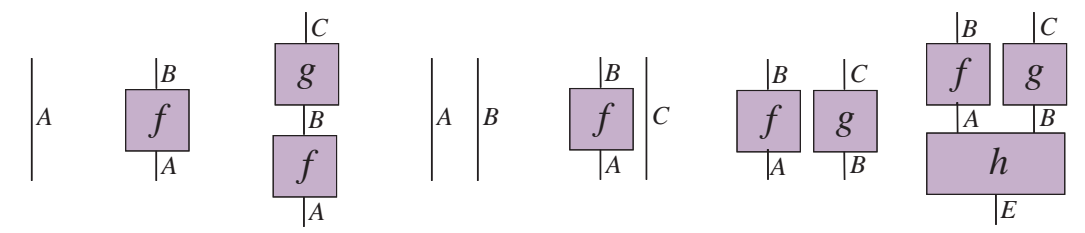

(The convention in these diagrams is that the 'upward' vertical direction represents progress of time.) A special role is played by boxes with either no input or no output, called states and costates respectively (cf. Dirac's kets and bras [19]) which we depict by triangles. Finally, we also need to consider diamonds which arise by post-composing a state with a matching costate (cf. inner-product or Dirac's bra-ket): 


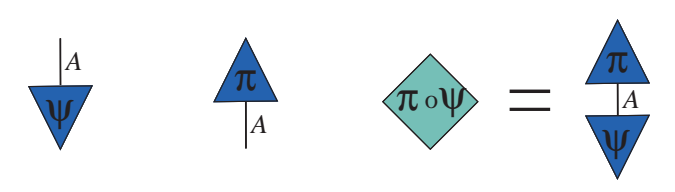

that is, algebraically,

$$
\psi: \mathrm{I} \rightarrow A \quad \pi: A \rightarrow \mathrm{I} \quad \pi \circ \psi: \mathrm{I} \rightarrow \mathrm{I}
$$

where $\mathrm{I}$ is the tensor unit: $A \otimes \mathrm{I} \simeq A \simeq \mathrm{I} \otimes A$. Extra structure is represented by (i) assigning a direction to the wires, where reversal of this direction is denoted by $A \mapsto A^{*}$, (ii) allowing reversal of boxes (cf. the adjoint for vector spaces), and, (iii) assuming that for each type $A$ there exists a special bipartite Bell-state and its adjoint Bell-costate:

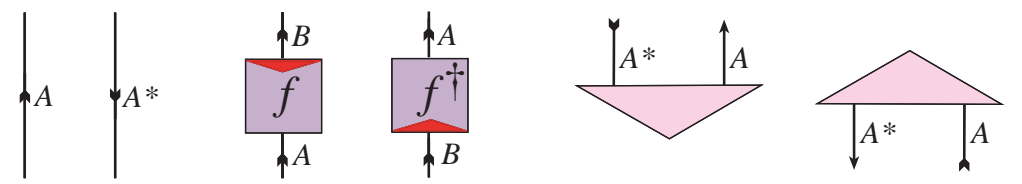

that is, algebraically,

$$
A \quad A^{*} \quad f: A \rightarrow B \quad f^{\dagger}: B \rightarrow A \quad \eta_{A}: \mathrm{I} \rightarrow A^{*} \otimes A \quad \eta_{A}^{\dagger}: A^{*} \otimes A \rightarrow \mathrm{I} .
$$

Hence, bras and kets are adjoint and the inner product has the form $(-)^{\dagger} \circ(-)$ on states. Essentially the sole axiom we impose is:

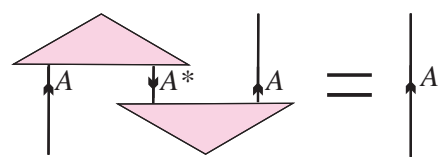

that is, algebraically,

$$
\left(\eta_{A^{*}}^{\dagger} \otimes 1_{A}\right) \circ\left(1_{A} \otimes \eta_{A}\right)=1_{A} .
$$

If we extend the graphical notation of Bell-(co)states to:
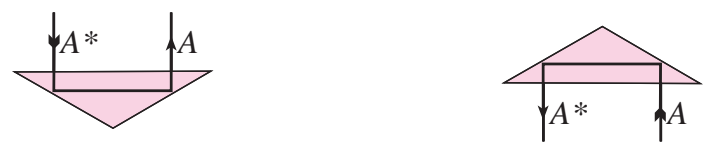

we obtain a clear graphical interpretation for the axiom:

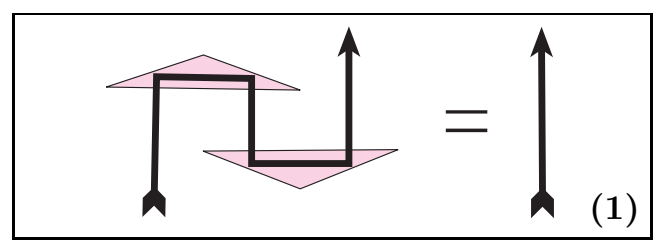


which now tells us that we are allowed to yank the black line straight:

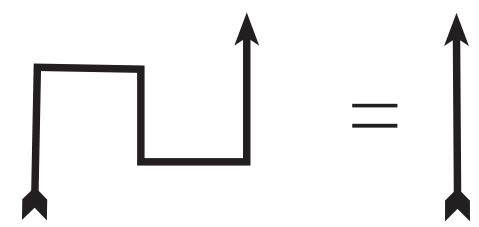

This equation and its diagrammatic counterpart should of course be compared to equation (2), and equation (1) and its accompanying diagram, in Section 3- they are one and the same, subject to minor differences in diagrammatic conventions.

This intuitive graphical calculus is an important benefit of the categorical axiomatics. Other advantages can be found in $[4,2]$.

\subsection{Quantum non-logic vs. quantum hyper-logic}

The term quantum logic is usually understood in connection with the 1936 Birkhoff-von Neumann proposal $[11,41]$ to consider the (closed) linear subspaces of a Hilbert space ordered by inclusion as the formal expression of the logical distinction between quantum and classical physics. While in classical logic we have deduction, the linear subspaces of a Hilbert space form a non-distributive lattice and hence there is no obvious notion of implication or deduction. Quantum logic was therefore always seen as logically very weak, or even as a non-logic. In addition, it has never given a satisfactory account of compound systems and entanglement.

On the other hand, compact closed logic in a sense goes beyond ordinary logic in the principles it admits. Indeed, while in ordinary categorical logic "logical deduction" implies that morphisms internalize as elements (which above we referred to above as states) i.e.

$$
B \stackrel{f}{\longrightarrow} C \stackrel{\simeq}{\longrightarrow} I \stackrel{\lceil f\rceil}{\longrightarrow} B \Rightarrow C
$$

(where $I$ is the tensor unit), in compact closed logic they internalize both as states and as costates, i.e.

$$
A \otimes B^{*} \stackrel{\lfloor f\rfloor}{\longrightarrow} I \stackrel{\simeq}{\longrightarrow} A \stackrel{f}{\longrightarrow} B \stackrel{\simeq}{\longrightarrow} I \stackrel{\lceil f\rceil}{\longrightarrow} A^{*} \otimes B
$$

where we introduce the following notation:

$\ulcorner f\urcorner=\left(1_{A^{*}} \otimes f\right) \circ \eta_{A}: I \rightarrow A^{*} \otimes B \quad\llcorner f\lrcorner=\epsilon_{B} \circ\left(f \otimes 1_{B^{*}}\right): A \otimes B^{*} \rightarrow I$. 
It is exactly this dual internalization which allows the straightening axiom in picture (1) to be expressed. In the graphical calculus this is witnessed by the fact that we can define both a state and a costate

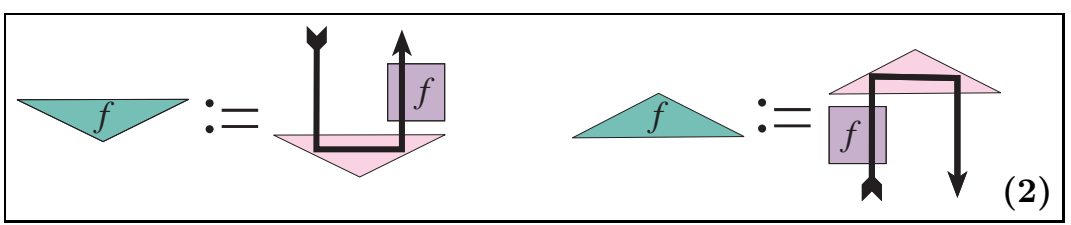

for each operation $f$. Physically, costates form the (destructive parts of) projectors, i.e. branches of projective measurements.

\subsubsection{Compositionality.}

The semantics is obviously compositional, both with respect to sequential composition of operations and parallel composition of types and operations, allowing the description of systems to be built up from smaller components. But we also have something more specific in mind: a form of compositionality with direct applications to the analysis of compound entangled systems. Since we have:

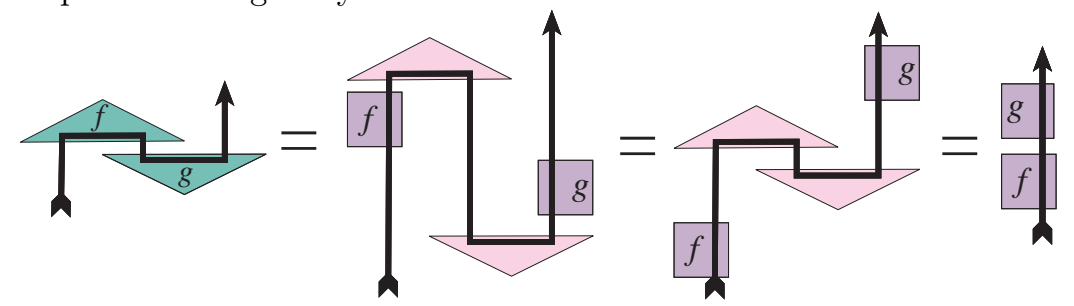

we obtain:

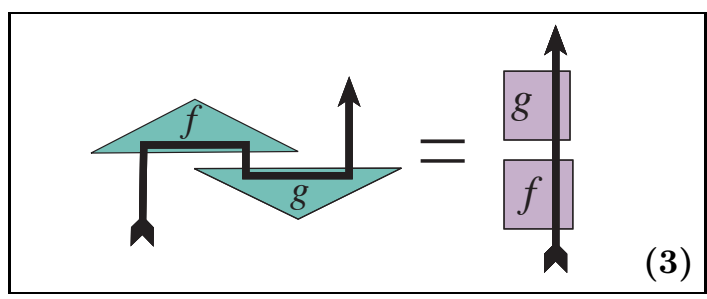

i.e. composition of operations can be internalized in the behavior of entangled states and costates. Note in particular the interesting phenomenon of "apparant reversal of the causal order" which is the source of many quite mystical interpretations of quantum teleportation in terms 
of "traveling backward in time" — cf. [35]. Indeed, while on the left, physically, we first prepare the state labeled $g$ and then apply the costate labeled $f$, the global effect is as if we first applied $f$ itself first, and only then $g$.

\subsubsection{Derivation of quantum teleportation.}

This is the most basic application of compositionality in action. Immediately from picture (1) we can read the quantum mechanical potential for teleportation:

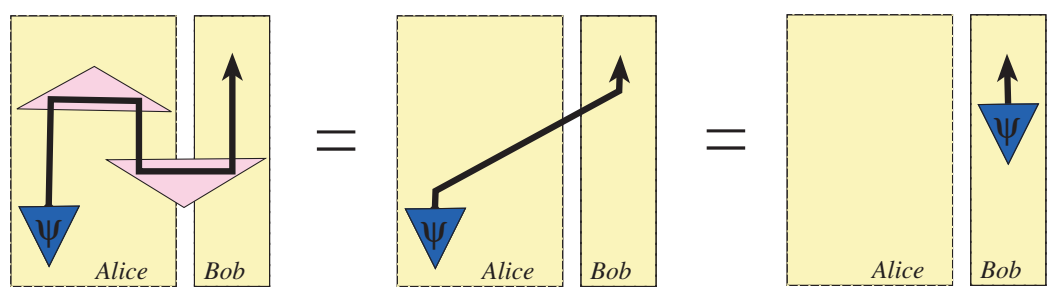

This is not quite the whole story, because of the non-deterministic nature of measurements. But it suffices to introduce a unitary correction. Using picture (3) the full description of teleportation becomes:

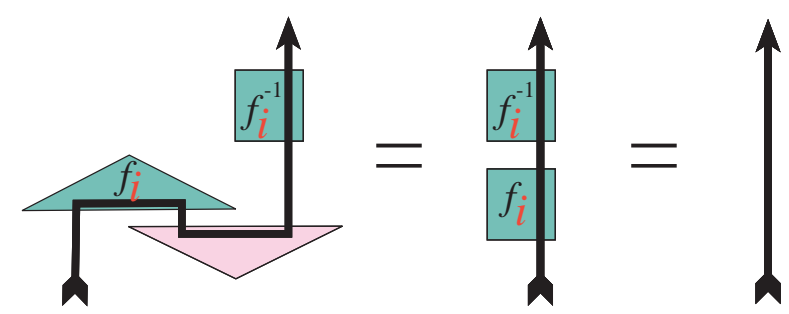

where the classical communication is now implicit in the fact that the index $i$ is both present in the costate (= measurement-branch) and the correction, and hence needs to be sent from Alice to Bob.

The classical communication can be made explicit as a fully fledged part of the formalism, using additional types: biproducts in [4], and "classical objects" in [17]. This allows entire protocols, including the interplay between quantum and classical information which is often their most subtle ingredient, to be captured and reasoned about rigorously in a single formal framework. 


\subsection{Remarks}

We close this Section with some remarks. We have seen that the categorical and diagrammatic setting for Quantum Mechanics developed by Abramsky and Coecke is strikingly close to that in which the TemperleyLieb category lives. The main difference is the free recourse to symmetry allowed in the Abramsky-Coecke setting (and in the main intended models for that setting, namely finite-dimensional Hilbert spaces with linear or completely positive maps). However, it is interesting to note that in the various protocols and constructions in Quantum Information and Computation which have been modelled in that setting to date [4], the symmetry has not played an essential role. The example of teleportation given above serves as an example.

This raises some natural questions:

\section{How much of QM/QIC lives in the plane?}

More precisely:

- Which protocols make essential use of symmetry?

- How much computational or information-processing power does the non-symmetric calculus have?

- Does braiding have some computational significance? (Remembering that between pivotal and symmetric we have braided strongly compact closed categories) [21].

\section{Planar Geometry of Interaction and the Temperley- Lieb Algebra}

We now address the issue of giving what, so far as I know, is the first direct - or "fully abstract" — description of the Temperley-Lieb category. Since the category $\mathcal{T}$ is directly and simply described as the free $R$-linear category generated by $\mathcal{D}$, we focus on the direct description of $\mathcal{D}$.

\section{Previous descriptions:}

- Algebraic, by generators and relations - whether "locally", of the Temperley-Lieb algebras $\mathcal{A}_{n}(\tau)$, as in Jones' presentation, or "globally", by a description of $\mathcal{D}$ as the free pivotal category, as in Proposition 1.1. 
- Kauffman's topological description: diagrams "up to planar isotopy".

In fact, it is well known (see e.g. [33]) that the diagrams are completely characterized by how the dots are joined up - i.e. by discrete relations on finite sets. This leaves us with the problem of how to capture

1. Planarity

2. The multiplication of diagrams - i.e. composition in $\mathcal{D}$

purely in terms of the data given by these relations.

The answers to these questions exhibit the connections that exist between the Temperley-Lieb category and what is commonly known as the "Geometry of Interaction". This is a dynamical/geometrical interpretation of proofs and Cut Elimination initiated by Girard [23] as an off-shoot of Linear Logic [22]. The general setting for these notions is now known to be that of traced monoidal and compact closed categories - in particular, in the free construction of compact closed categories over traced monoidal categories $[1,7]$. In fact, this general construction was first clearly described in [27], where one of the leading motivations was the knot-theoretic context.

Our results in this Section establish a two-way connection. In one direction, we shall use ideas from Geometry of Interaction to answer Question 2 above: that is, to define path composition (including the formation of loops) purely in terms of the discrete relations tabulating how the dots are joined up. In the other direction, our answer to Question 1 will allow us to consider a natural planar variant of the Geometry of Interaction.

\subsection{Some preliminary notions}

\subsubsection{Partial Orders}

We use the notation $P=\left(|P|, \leq_{P}\right)$ for partial orders. Thus $|P|$ is the underlying set, and $\leq_{P}$ is the order relation (reflexive, transitive and antisymmetric) on this set. An order relation is linear if for all $x, y \in|P|, x \leq_{P} y$ or $y \leq_{P} x$.

Given a natural number $n$, we define $[n]:=\{1<\cdots<n\}$, the linear order of length $n$. We define several constructions on partial orders. Given partial orders $P, Q$, we define: 
- The disjoint sum $P \oplus Q$, where $|P \oplus Q|=|P|+|Q|$, the disjoint union of $|P|$ and $|Q|$, and

$$
x \leq_{P \oplus Q} y \Longleftrightarrow\left(x \leq_{P} y\right) \vee\left(x \leq_{Q} y\right) .
$$

- The concatenation $P \triangleleft Q$, where $|P \triangleleft Q|=|P|+|Q|$, with the following order:

$$
x \leq_{P \triangleleft Q} y \Longleftrightarrow\left(x \leq_{P} y\right) \vee\left(x \leq_{Q} y\right) \vee(x \in P \wedge y \in Q) .
$$

- $P^{\mathrm{op}}=\left(|P|, \geq_{P}\right)$.

Given elements $x, y$ of a partial order $P$, we define:

$$
\begin{array}{rlc}
x \uparrow y & \Leftrightarrow & \left(x \leq_{P} y\right) \vee\left(y \leq_{P} x\right) \\
x \# y & \Leftrightarrow & \neg(x \uparrow y) .
\end{array}
$$

\subsubsection{Relations}

A relation on a set $X$ is a subset of the cartesian product: $R \subseteq X \times X$. Since relations are sets, they are closed under unions and intersections. We shall also use the following operations of relation algebra:

\section{Identity relation:}

Relation composition:

Relational converse:

Transitive closure:

Reflexive transitive closure: $R^{*}:=\bigcup_{k \geq 0} R^{k}$

Here $R^{k}$ is defined inductively: $R^{0}:=1_{X}, R^{1}:=R, R^{k+1}:=R ; R^{k}$. A relation $R$ is single-valued or a partial function if $R^{c} ; R \subseteq 1_{X}$. It is total if $R ; R^{c} \supseteq 1_{X}$. A function $f: X \rightarrow X$ is a single-valued, total relation.

These notions extend naturally to relations $R \subseteq X \times Y$.

\subsubsection{Involutions}

A fixed-point free involution on a set $X$ is a function $f: X \rightarrow X$ such that

$$
f^{2}=1_{X}, \quad f \cap 1_{X}=\varnothing .
$$


Thus for such a function $f(x)=y \Leftrightarrow x=f(y)$ and $f(x) \neq x$. We write $\operatorname{lnv}(X)$ for the set of fixed-point free involutions on a set $X$. Note that $\operatorname{lnv}(X)$ is not closed under function composition; nor does it contain the identity function. We must look elsewhere for suitable notions of composition and identity.

An involution is equivalently described as a parition of $X$ into 2element subsets:

$$
X=\bigcup E, \quad \text { where } E=\{\{x, y\} \mid f(x)=y\} .
$$

This defines an undirected graph $G_{f}=(X, E)$. Clearly $G_{f}$ is 1-regular [18]: each vertex has exactly one incident edge. Conversely, every graph $G=(X, E)$ with this property determines a unique $f \in \operatorname{lnv}(X)$ with $G_{f}=G$. Note that a finite set can only carry such a structure of its cardinality is even.

\subsection{Formalizing diagrams}

From our previous discussion, it is fairly clear how we will proceed to formalize morphisms $\mathbf{n} \rightarrow \mathbf{m}$ in $\mathcal{D}$. Given $n, m \in \mathbb{N}$, we define $\mathbf{N}(n, m)=$ $[n] \oplus[m]$. We visualize this partial order as

$$
\begin{array}{llll}
1 & 2 & \ldots & n \\
\hdashline & \bullet & \cdots & 0
\end{array}
$$

$$
\begin{array}{lllll}
1^{\prime} & \stackrel{0}{2^{\prime}} & \cdots & \cdots & \bullet \\
m^{\prime}
\end{array}
$$

We use the notation $i^{\prime}$ to distinguish the elements of $[m]$ in this disjoint union from those of $[n]$, which are unprimed. Note that the order on $\mathrm{N}(n, m)$ has an immediate spatial interpretation in the diagrammatic representation: $i<j$ just in case $i$ lies to the left of $j$ on either the top or bottom line of dots, corresponding to $[n]$ and $[m]$ respectively.

A diagram connecting up dots pairwise will be formalized as a map $f \in \operatorname{Inv}(|\mathrm{N}(n, m)|)$. Such a map can be visualized by drawing undirected arcs between the pairs of nodes $i, j$ such that $f(i)=j$. 


\subsubsection{Example}

The map $f \in \operatorname{lnv}(|\mathrm{N}(4,2)|)$ such that

$$
f: 1 \leftrightarrow 2^{\prime}, \quad 2 \leftrightarrow 4, \quad 3 \leftrightarrow 1^{\prime}
$$

is depicted thus:

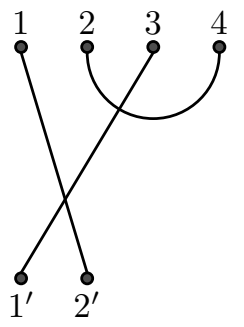

Our task is now is characterize those involutions which are planar. The key idea is that this can be done using just the order relations we have introduced.

\subsection{Characterizing Planarity}

A map $f \in \operatorname{Inv}(|\mathrm{N}(n, m)|)$ will be called planar if it satisfies the following two conditions, for all $i, j \in \mathrm{N}(n, m)$ :

$$
\begin{aligned}
& \text { (PL1) } \quad i<j<f(i) \quad \Longrightarrow i<f(j)<f(i) \\
& \text { (PL2) } f(i) \# i<j \# f(j) \Longrightarrow \quad f(i)<f(j) .
\end{aligned}
$$

It is instructive to see which possibilities are excluded by these conditions.

\subsubsection{First condition}

$$
(\mathrm{PL} 1) i<j<f(i) \Longrightarrow i<f(j)<f(i)
$$

(PL1) rules out

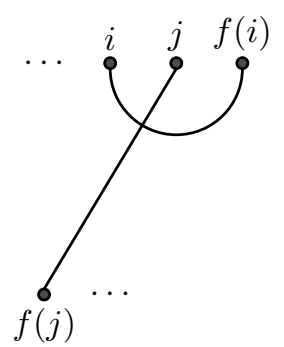


where $f(j) \# f(i)$, and also

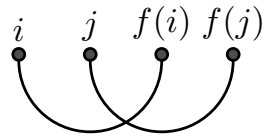

where $f(i)<f(j)$.

\subsubsection{Second condition}

$$
\text { (PL2) } f(i) \# i<j \# f(j) \Longrightarrow f(i)<f(j) \text {. }
$$

Similarly, (PL2) rules out

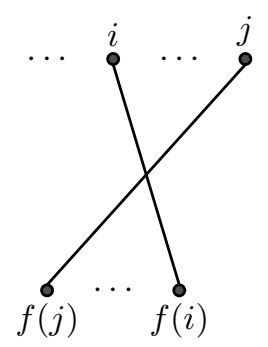

We write $\mathcal{P}(n, m)$ for the set of planar maps in $\operatorname{lnv}(|\mathrm{N}(n, m)|)$.

\section{PROPOSITION 1.6}

1. Every planar diagram satisfies the two conditions.

2. Every involution satisfying the two conditions can be drawn as a planar diagram.

Rather than proving this directly, it is simpler, and also instructive, to reduce it to a special case. We consider arrows in $\mathcal{D}$ of the special form $I \rightarrow \mathbf{n}$. Such arrows consist only of caps. They correspond to points, or states in the terminology of Section 5.

Since the top row of dots is empty, in this case we have a linear order, and the premise of condition (PL2) can never arise. Hence planarity for such arrows is just the simple condition (PL1) - which can be seen to be equivalent to saying that, if we write a left parenthesis for each left end of a cap, and a right parenthesis for each right end, we get a well-formed string of parentheses. Thus 


\section{$\Omega \Omega$}

corresponds to

$$
()(()) \text {. }
$$

(Of course, exactly similar comments apply to arrows of the form $\mathbf{n} \rightarrow I$, i.e. costates.) It is also clear ${ }^{5}$ that Proposition 1.6 holds for such arrows.

Now we recall that quite generally, in any pivotal category we have the Hom-Tensor adjunction

$$
A \otimes B^{*} \stackrel{\lfloor f\rfloor}{\longrightarrow} I \stackrel{\simeq}{\longrightarrow} A \stackrel{f}{\longrightarrow} B \stackrel{\simeq}{\longrightarrow} I \stackrel{\lceil f\rceil}{\longrightarrow} A^{*} \otimes B
$$

$\ulcorner f\urcorner=\left(1_{A^{*}} \otimes f\right) \circ \eta_{A}: I \rightarrow A^{*} \otimes B \quad\llcorner f\lrcorner=\epsilon_{B} \circ\left(f \otimes 1_{B^{*}}\right): A \otimes B^{*} \rightarrow I$.

We call $\ulcorner f\urcorner$ the name of $f$, and $\llcorner f\lrcorner$ the coname. The inverse to the map $f \mapsto\ulcorner f\urcorner$ is defined by

$$
g: I \rightarrow A^{*} \otimes B \mapsto\left(\epsilon_{A} \otimes 1_{B}\right) \circ\left(1_{A} \otimes g\right): A \rightarrow B .
$$

For example, we compute the name of the left wave:

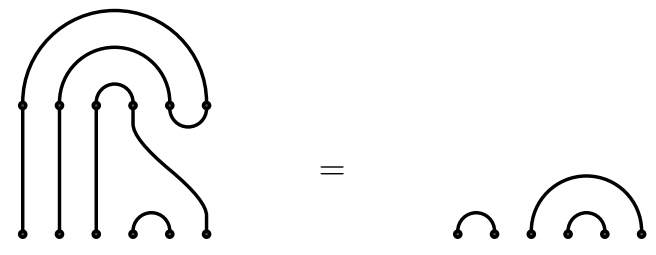

Applying the inverse transformation:

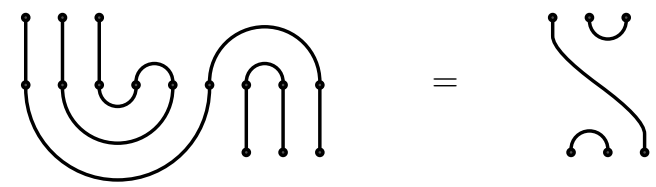

Note also that the unit is the name of the identity: $\eta_{\mathbf{n}}=\left\ulcorner 1_{\mathbf{n}}\right\urcorner$, and similarly $\epsilon_{\mathbf{n}}=\left\llcorner 1_{\mathbf{n}}\right\lrcorner$.

Thus we see that diagrammatically, the process of forming the name of an arrow involves reversing the left-right order of the top row of dots by rotating them concentrically, and sliding them down to lie parallel with, and to the left of, the bottom row. In this process, cups are turned

${ }^{5}$ With an implicit appeal to the Jordan Curve Theorem! 
into caps, while through lines are stretched out and turned to also form caps.

This transposition of the top row of dots can be described ordertheoretically, as replacing the partial order $[n] \oplus[m]$ by the linear order $[n]^{\mathrm{op}} \triangleleft[m]$. Note that the underlying sets of these two partial orders are the same: $|[n] \oplus[m]|=\left|[n]^{\text {op }} \triangleleft[m]\right|$. Thus $\ulcorner f\urcorner$ is essentially the same function as $f$.

\section{PROPOSITION 1.7}

For $f \in \operatorname{Inv}(|\mathrm{N}(n, m)|)$, the following are equivalent:

1. $f$ satisfies (PL1) and (PL2) with respect to $[n] \oplus[m]$.

2. $f$ satisfies (PL1) with respect to $[n]^{\mathrm{op}} \triangleleft[m]$.

Proof Firstly, assume (2), and suppose $f(i) \# i<j \# f(j)$. If $i<j$ in the bottom row, then $f(j)<i<j$ in $[n]^{\text {op }} \triangleleft[m]$, so by (PL1), $f(j)<f(i)<j$, i.e. $f(i)<f(j)$ in $[n] \oplus[m]$, as required. Now suppose $i<j$ in the top row. Then $j<i<f(j)$ in $[n]^{\text {op }} \triangleleft[m]$, so by (PL1), $j<f(i)<f(j)$, and in particular $f(i)<f(j)$.

Now assume (1), and suppose that $i<j<f(i)$ in $[n]^{\text {op }} \triangleleft[m]$. The interesting case is where $i$ is in the top row and $f(i)$ in the bottom row. We need to do some case analysis. Suppose firstly that $j$ is in the top row. If $f(j)$ is in the bottom row, then $f(j) \# j<i \# f(i)$ in $[n] \oplus[m]$, and we can apply (PL2) to conclude that $f(j)<f(i)$, and hence $i<f(j)<f(i)$ in $[n]^{\text {op }} \triangleleft[m]$. If $f(j)$ is in the top row, we must have $f(j)<i$ by (PL1) for $[n] \oplus[m]$, and hence $i<f(j)<f(i)$ in $[n]^{\text {op }} \triangleleft[m]$.

Now suppose that $j$ is in the bottom row. If $f(j)$ is in the bottom row, we must have $f(j)<f(i)$ by (PL1). If $f(j)$ is in the top row, then we have $f(j) \# j<f(i) \# i$ in $[n] \oplus[m]$, so by (PL2) we have $f(j)<i$, and hence $i<f(j)<f(i)$ in $[n]^{\text {op }} \triangleleft[m]$.

Since (PL1) characterizes planarity for $\ulcorner f\urcorner$, it follows that (PL1) and (PL2) characterize planarity for $f$.

\subsection{The Temperley-Lieb Category}

Our aim is now to define a category $\mathbf{T L}$, which will yield the desired description of the diagrammatic category $\mathcal{D}$. The objects of TL are the natural numbers. The homset $\mathbf{T L}(\mathbf{n}, \mathbf{m})$ is defined to be the cartesian product $\mathbb{N} \times \mathcal{P}(n, m)$. Thus a morphism $\mathbf{n} \rightarrow \mathbf{m}$ in $\mathbf{T L}$ consists of a pair 
$(k, f)$, where $k$ is a natural number, and $f \in \mathcal{P}(n, m)$ is a planar map in $\operatorname{lnv}(|\mathrm{N}(n, m)|)$. The idea is that $k$ is a counter for the number of loops, so such an arrow can be written $\delta^{k} \cdot f$ in the notation used previously.

It remains to define the composition and identities in this category. Clearly (even leaving aside the natural number components of morphisms) composition cannot be defined as ordinary function composition. This does not even make sense - the codomain of an involution $f \in \mathcal{P}(n, m)$ does not match the domain of an involution $g \in \mathcal{P}(m, p)$ let alone yield a function with the necessary properties to be a morphism in the category.

\subsubsection{Composition: The "Execution Formula"}

Consider a map $f:[n]+[m] \longrightarrow[n]+[m]$. Each input lies in either $[n]$ or $[m]$ (exclusive or), and similarly for the corresponding output. This leads to a decomposition of $f$ into four disjoint partial maps:

$$
\begin{array}{ll}
f_{n, n}:[n] \longrightarrow[n] & f_{n, m}:[n] \longrightarrow[m] \\
f_{m, n}:[m] \longrightarrow[n] & f_{m, m}:[m] \longrightarrow[m]
\end{array}
$$

so that $f$ can be recovered as the disjoint union of these four maps. If $f$ is an involution, then these maps will be partial involutions.

Note that these components have a natural diagrammatic reading: $f_{n, n}$ describes the cups of $f, f_{m, m}$ the caps, and $f_{n, m}=f_{m, n}^{c}$ the through lines.

Now suppose we have maps $f:[n]+[m] \rightarrow[n]+[m]$ and $g:[m]+[p] \rightarrow$ $[m]+[p]$. We write the decompositions of $f$ and $g$ as above in matrix form:

$$
f=\left(\begin{array}{cc}
f_{n, n} & f_{n, m} \\
f_{m, n} & f_{m, m}
\end{array}\right) \quad g=\left(\begin{array}{cc}
g_{m, m} & g_{m, p} \\
g_{p, m} & g_{p, p}
\end{array}\right)
$$

We can view these maps as binary relations on $[n]+[m]$ and $[m]+[p]$ respectively, and use relational algebra (union $R \cup S$, relational composition $R ; S$ and reflexive transitive closure $R^{*}$ ) to define a new relation $\theta$ on $[n]+[p]$. If we write

$$
\theta=\left(\begin{array}{cc}
\theta_{n, n} & \theta_{n, p} \\
\theta_{p, n} & \theta_{p, p}
\end{array}\right)
$$


so that $\theta$ is the disjoint union of these four components, then we can define it component-wise as follows:

$$
\begin{aligned}
& \theta_{n, n}=f_{n, n} \cup f_{n, m} ; g_{m, m} ;\left(f_{m, m} ; g_{m, m}\right)^{*} ; f_{m, n} \\
& \theta_{n, p}=f_{n, m} ;\left(g_{m, m} ; f_{m, m}\right)^{*} ; g_{m, p} \\
& \theta_{p, n}=g_{p, m} ;\left(f_{m, m} ; g_{m, m}\right)^{*} ; f_{m, n} \\
& \theta_{p, p}=g_{p, p} \cup g_{p, m} ; f_{m, m} ;\left(g_{m, m} ; f_{m, m}\right)^{*} ; g_{m, p} .
\end{aligned}
$$

We can give clear intuitive readings for how these formulas express composition of paths in diagrams in terms of relational algebra:

- The component $\theta_{n, n}$ describes the cups of the diagram resulting from the composition. These are the union of the cups of $f\left(f_{n, n}\right)$, together with paths that start from the top row with a through line of $f$, given by $f_{n, m}$, then go through an alternating odd-length sequence of cups of $g\left(g_{m, m}\right)$ and caps of $f\left(f_{m, m}\right)$, and finally return to the top row by a through line of $f\left(f_{m, n}\right)$.

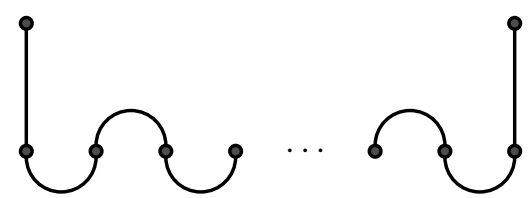

- Similarly, $\theta_{p, p}$ describes the caps of the composition.

- $\theta_{n, p}=\theta_{p, n}^{c}$ describe the through lines. Thus $\theta_{n, p}$ describes paths which start with a through line of $f$ from $n$ to $m$, continue with an alternating even-length (and possibly empty) sequence of cups of $g$ and caps of $f$, and finish with a through line of $g$ from $m$ to $p$.

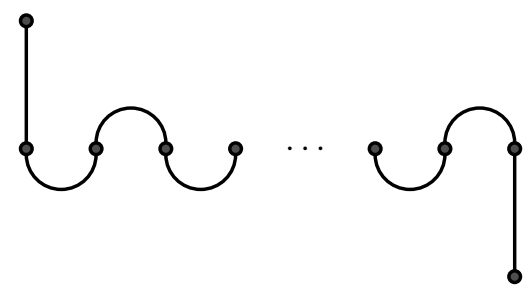

All through lines from $n$ to $p$ must have this form. 
This formula corresponds to the interpretation of Cut-Elimination in the Geometry of Interaction interpretation of proofs in Linear Logic (and by extension in related logics and type theories) [23]. A more abstract and general perspective on how this construction arises can be given in the setting of traced monoidal categories [27, 1].

\section{PROPOSITION 1.8}

If $f$ and $g$ are planar, so is $\theta$.

We write $\theta=g \odot f \in \mathcal{P}(n, p)$

\subsubsection{Cycles}

Given $f \in \mathcal{P}(n, m), g \in \mathcal{P}(m, p)$, we define $\chi(f, g):=f_{m, m} ; g_{m, m}$. Note that $\chi(f, g)^{c}=\left(g_{m, m} ; f_{m, m}\right)$, and

$$
\chi(f, g) ; \chi(f, g)^{c} \subseteq 1_{[m]}, \quad \chi(f, g)^{c} ; \chi(f, g) \subseteq 1_{[m]} .
$$

Thus $\chi(f, g)$ is a partial bijection. However, in general it is neither an involution, nor fixpoint-free. The cyclic elements of $\chi(f, g)$ are those elements of $[m]$ which lie in the intersection

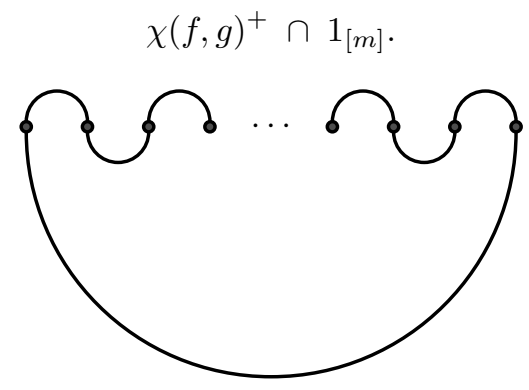

Thus if $i$ is a cyclic element, there is a least $k>0$ such that $\chi(f, g)^{k}(i)=$ $i$. The corresponding cycle is

$$
\left\{i, \chi(f, g)(i), \ldots, \chi(f, g)^{k-1}(i)\right\} .
$$

Distinct cycles are disjoint. We write $Z(f, g)$ for the number of distinct cycles of $\chi(f, g)$. 


\subsubsection{Composition and Identities}

Finally, we define the composition of morphisms in TL. Given $(s, f)$ : $\mathbf{n} \rightarrow \mathbf{m}$ and $(t, g): \mathbf{m} \rightarrow \mathbf{p}:$

$$
(t, g) \circ(s, f)=(s+t+Z(f, g), g \odot f) .
$$

The identity morphism $\mathrm{id}_{\mathbf{n}}: \mathbf{n} \rightarrow \mathbf{n}$ is defined to be the pair $\left(0, \tau_{n, n}\right)$, where $\tau_{n, n}$ is the twist map on $[n]+[n]$; i.e. the involution $i \leftrightarrow i^{\prime}$. Diagrammatically, this is just

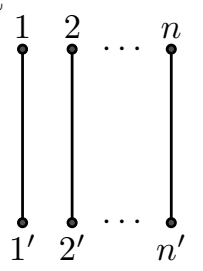

Note that this is not the identity map on $[n]+[n]$ - indeed it is (necessarily) fixpoint free!

\section{PROPOSITION 1.9}

TL with composition and identities as defined above is a category.

\subsubsection{TL as a pivotal category}

The monoidal structure of TL is straightforward. If $(k, f): \mathbf{n} \rightarrow \mathbf{m}$ and $(l, g): \mathbf{p} \rightarrow \mathbf{q}$, then $(k+l, f+g): \mathbf{n}+\mathbf{p} \rightarrow \mathbf{m}+\mathbf{q}$, where $f+g \in$ $\mathcal{P}(n+p, m+q)$ is the evident disjoint union of the involutions $f$ and $g$.

The unit $\eta_{\mathbf{n}}: I \rightarrow \mathbf{n}+\mathbf{n}$ is given by

$$
i \leftrightarrow i^{\prime} \quad(1 \leq i \leq n),
$$

and similarly for the counit. Note that identities, units and counits are all essentially the same maps, but with distinct types, which partition their arguments between inputs and outputs differently.

We describe the dual, adjoint and conjugate of an arrow $(k, f): \mathbf{n} \rightarrow$ m. Let $\tau_{n, m}:[n]+[m] \stackrel{\cong}{\longrightarrow}[m]+[n]$ be the symmetry isomorphism of the disjoint union, and

$$
\rho_{n}:[n] \stackrel{\cong}{\longrightarrow}[n]:: i \mapsto n-i+1
$$

be the order-reversal isomorphism. Note that

$$
\tau_{n, m}^{-1}=\tau_{m, n}, \quad \rho_{n}^{-1}=\rho_{n}, \quad \tau_{n, m} \circ\left(\rho_{n}+\rho_{m}\right)=\left(\rho_{m}+\rho_{n}\right) \circ \tau_{n, m} .
$$


Then we have $(k, f)^{\bullet}=\left(k, f^{\bullet}\right)$, where:

$$
\begin{aligned}
& f^{\dagger}=\tau_{n . m} \circ f \circ \tau_{n, m}^{-1} \\
& f_{*}=\left(\rho_{n}+\rho_{m}\right) \circ f \circ\left(\rho_{n}+\rho_{m}\right)^{-1} \\
& f^{*}=\left(f^{\dagger}\right)_{*} .
\end{aligned}
$$

\subsubsection{The Main Result}

\section{THEOREM 1.1}

TL is isomorphic as a strict, pivotal dagger category to $\mathcal{D}$.

As an immediate Corollary of this result and Proposition 1.2, we have:

\section{THEOREM 1.2}

TL is the free strict, pivotal dagger category on one self-dual generator.

This is in the same spirit as the characterizations of free compact and dagger compact categories in $[34,3]$.

These results can easily be extended to descriptions of the free pivotal dagger category over an arbitrary generating category, leading to oriented Temperley-Lieb algebras with primitive (physical) operations. We refer to [3] for a more detailed presentation (in the symmetric case).

\section{$7 \quad$ Planar $\lambda$-Calculus}

Our aim in this section is to show how a restricted form of $\lambda$-calculus can be interpreted in the Temperley-Lieb category, and how $\beta$-reduction of $\lambda$-terms, which is an important foundational paradigm for computation, is then reflected diagrammatically as geometric simplification, i.e. "yanking lines straight". We can give only a brief indication of what is in fact a rich topic in its own right. See $[6,7,31]$ for discussions of related matters. 


\subsection{The $\lambda$-Calculus}

We begin with a (very) brief review of the $\lambda$-calculus $[14,9]$, which is an important foundational paradigm in Logic and Computation, and in particular forms the basis for all modern functional programming languages.

The syntax of the $\lambda$-calculus is beguilingly simple. Given a set of variables $x, y, z, \ldots$ we define the set of terms as follows:

$$
t::=x|\underbrace{t u}_{\text {application }}| \underbrace{\lambda x \cdot t}_{\text {abstraction }}
$$

Notational Convention: We write

$$
t_{1} t_{2} \cdots t_{k} \equiv\left(\cdots\left(t_{1} t_{2}\right) \cdots\right) t_{k}
$$

Some examples of terms:

\begin{tabular}{ll}
\hline$\lambda x \cdot x$ & identity function \\
$\lambda f \cdot \lambda x \cdot f x$ & application \\
$\lambda f \cdot \lambda x \cdot f(f x)$ & double application \\
$\lambda f \cdot \lambda g \cdot \lambda x \cdot g(f(x))$ & composition $g \circ f$ \\
\hline
\end{tabular}

The basic equation governing this calculus is $\beta$-conversion:

$$
(\lambda x . t) u=t[u / x]
$$

E.g. (assuming some arithmetic operations are given)

$$
(\lambda f \cdot \lambda x \cdot f(f x))(\lambda x \cdot x+1) 0=(0+1)+1=2 .
$$

By orienting this equation, we get a 'dynamics' $-\beta$-reduction

$$
(\lambda x . t) u \rightarrow t[u / x]
$$

Despite its sparse syntax, $\lambda$-calculus is very expressive-it is in fact a universal model of computation, equivalent to Turing machines.

\subsection{Types}

One important way of constraining the $\lambda$-calculus is to introduce Types.

Types are there to stop you doing (bad) things 
Types are in fact one of the most fruitful positive ideas in Computer Science!

We shall introduce a (highly restrictive) type system, such that the typable terms can be interpreted in the Temperley-Lieb category (in fact, in any pivotal category).

Firstly, assuming some set of basic types $B$, we define a syntax of general types:

$$
T::=B \mid T \rightarrow T .
$$

Intuitively, $T \rightarrow U$ represents the type of functions which take inputs of type $T$ to outputs of type $U$.

Notational Convention: We write

$$
T_{1} \rightarrow T_{2} \rightarrow \cdots T_{k} \rightarrow T_{k+1} \quad \equiv \quad T_{1} \rightarrow\left(T_{2} \rightarrow \cdots\left(T_{k} \rightarrow T_{k+1}\right) \cdots\right) .
$$

Examples:

$$
\begin{array}{cc}
A \rightarrow A \rightarrow A & \text { first-order function type } \\
(A \rightarrow A) \rightarrow A & \text { second-order function type }
\end{array}
$$

We now introduce a formal system for deriving typing judgements, of the form:

$$
x_{1}: T_{1}, \ldots x_{k}: T_{k} \vdash t: T .
$$

Such a judgement asserts that the term $t$ has type $T$ under the assumption (or: in the context) that the variable $x_{1}$ has type $T_{1}, \ldots, x_{k}$ has type $T_{k}$. All the variables $x_{i}$ appearing in the context must be distinct - and in our setting, the order in which the variables appear in the list is significant.

There is one basic form of axiom, for typing variables:

\section{Variable}

$$
\overline{x: T \vdash x: T}
$$

and two inference rules, for typing abstractions and applications respectively:

\section{Function}

$$
\frac{\Gamma, x: U \vdash t: T}{\Gamma \vdash \lambda x . t: U \rightarrow T} \quad \frac{\Gamma \vdash t: U \rightarrow T \quad \Delta \vdash u: U}{\Gamma, \Delta \vdash t u: T}
$$

Note that $\Gamma, \Delta$ represents the concatenation of the lists $\Gamma, \Delta$. This implies that the variables appearing in $\Gamma$ and $\Delta$ are distinct - an important linearity constraint in the sense of Linear Logic [22]. 


\subsection{Interpretation in Pivotal Categories}

We now show how terms typable in our system can be interpreted in a pivotal category $\mathcal{C}$. We assume firstly that the basic types $B$ have been interpreted as objects $\llbracket B \rrbracket$ of $\mathcal{C}$. We then extend this to general types by:

$$
\llbracket T \rightarrow U \rrbracket=\llbracket U \rrbracket \otimes \llbracket T \rrbracket^{*} .
$$

Now we show how, for each typing judgement $\Gamma \vdash t: T$, to assign an arrow

$$
\llbracket \Gamma \rrbracket \longrightarrow \llbracket T \rrbracket,
$$

where if $\Gamma=x_{1}: T_{1}, \ldots x_{k}: T_{k}$,

$$
\llbracket \Gamma \rrbracket=\llbracket T_{1} \rrbracket \otimes \cdots \otimes \llbracket T_{k} \rrbracket .
$$

This assignment is defined by induction on the derivation of the typing judgement in the formal system.

Variable

$$
\overline{x: T \vdash x: T} \quad \overline{1_{\llbracket T \rrbracket}: \llbracket T \rrbracket \longrightarrow \llbracket T \rrbracket}
$$

\section{Abstraction}

To interpret $\lambda$-abstraction, we use the adjunction

$$
\begin{gathered}
\Lambda_{\mathrm{r}}: \mathcal{C}(A \otimes B, C) \simeq \mathcal{C}\left(A, C \otimes B^{*}\right) \\
\Lambda_{\mathrm{r}}(f)=A \stackrel{1_{A} \otimes \eta_{B *}}{\longrightarrow} A \otimes B \otimes B^{*} \stackrel{f \otimes 1_{B^{*}}}{\longrightarrow} C \otimes B^{*}
\end{gathered}
$$

We can then define:

$$
\frac{\Gamma, x: U \vdash t: T}{\Gamma \vdash \lambda x: U . t: U \rightarrow T} \quad \frac{\llbracket t \rrbracket: \llbracket \Gamma \rrbracket \otimes \llbracket U \rrbracket \longrightarrow \llbracket T \rrbracket}{\Lambda_{\mathrm{r}}(\llbracket t \rrbracket): \llbracket \Gamma \rrbracket \longrightarrow \llbracket T \rrbracket \otimes \llbracket U \rrbracket^{*}}
$$

\section{Application}

We use the following operation of right application:

$$
\begin{gathered}
\text { RApp : } \mathcal{C}\left(C, B \otimes A^{*}\right) \times \mathcal{C}(D, A) \longrightarrow \mathcal{C}(C \otimes D, B) \\
\operatorname{RApp}(f, g)=C \otimes D \stackrel{f \otimes g}{\longrightarrow} B \otimes A^{*} \otimes A \stackrel{1_{B} \otimes \epsilon_{B^{*}}}{\longrightarrow} B .
\end{gathered}
$$

We can then define:

$$
\frac{\Gamma \vdash t: U \rightarrow T \quad \Delta \vdash u: U}{\Gamma, \Delta \vdash t u: T} \quad \frac{\llbracket t \rrbracket: \llbracket \Gamma \rrbracket \longrightarrow \llbracket T \rrbracket \otimes \llbracket U \rrbracket^{*} \quad \llbracket u \rrbracket: \llbracket \Delta \rrbracket \longrightarrow \llbracket U \rrbracket}{\operatorname{RApp}(\llbracket t \rrbracket, \llbracket u \rrbracket): \llbracket \Gamma \rrbracket \otimes \llbracket \Delta \rrbracket \longrightarrow \llbracket T \rrbracket}
$$

It can be proved that this interpretation is sound for $\beta$-conversion, i.e.

$$
\llbracket(\lambda x . t) u \rrbracket=\llbracket t[u / x] \rrbracket
$$

in any pivotal category. 


\subsection{An Example}

We now discuss an example to show how all this works diagrammatically in TL. We shall consider the bracketing combinator

$$
\mathbf{B} \equiv \lambda x \cdot \lambda y \cdot \lambda z . x(y z) .
$$

This is characterized by the equation

$$
\mathbf{B} a b c=a(b c) .
$$

Firstly, we derive a typing judgement for this term:

$$
\begin{aligned}
& x: B \rightarrow C \vdash x: B \rightarrow C \quad \frac{y: A \rightarrow B \vdash y: A \rightarrow B \quad z: A \vdash z: A}{y: A \rightarrow B, z: A \vdash y z: B} \\
& \frac{\frac{x: B \rightarrow C, y: A \rightarrow B, z: A \vdash x(y z): C}{x: B \rightarrow C, y: A \rightarrow B \vdash \lambda z \cdot x(y z): A \rightarrow C}}{\frac{x: B \rightarrow C \vdash \lambda y \cdot \lambda z \cdot x(y z):(A \rightarrow B) \rightarrow(A \rightarrow C)}{\vdash \lambda x \cdot \lambda y \cdot \lambda z \cdot x(y z):(B \rightarrow C) \rightarrow(A \rightarrow B) \rightarrow(A \rightarrow C)}}
\end{aligned}
$$

Now we take $A=B=C=\mathbf{1}$ in TL. The interpretation of the open term

$$
x: B \rightarrow C, y: A \rightarrow B, z: A \vdash x(y z): C
$$

is as follows:

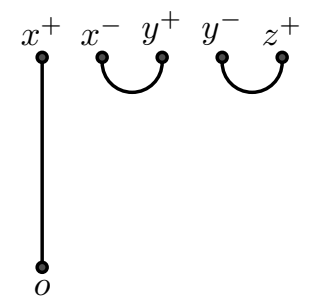

Here $x^{+}$is the output of $x$, and $x^{-}$the input, and similarly for $y$. The output of the whole expression is $o$. When we abstract the variables, we obtain the following caps-only diagram:

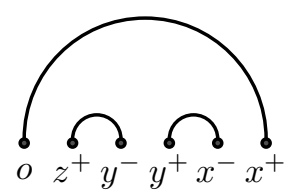

Now we consider an application $\mathbf{B} a b c$ : 


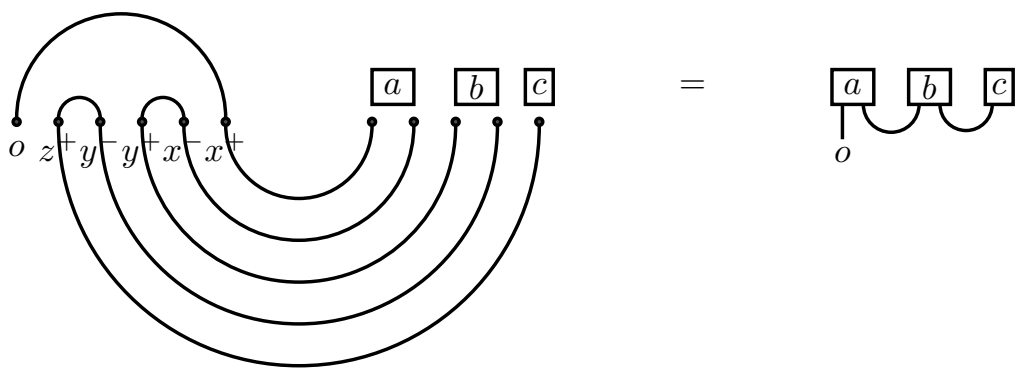

\subsection{Discussion}

The typed $\lambda$-calculus we have used here is in fact a fragment of the Lambek calculus [36], a basic non-commutative logic and $\lambda$-calculus, which has found extensive applications in computational linguistics [13, 38]. The Lambek calculus can be interpreted in any monoidal biclosed category, and has notions of left abstraction and application, as well as the right-handed versions we have described here. Pivotal categories have stronger properties than monoidal biclosure; for example, duality and adjoints allow the left- and right-handed versions of abstraction and application to be defined in terms of each other. Moreover, the duality means that the corresponding logic has a classical format, with an involutive negation. Thus there is much more to this topic than we have had the time to discuss here. We merely hope to have given an impression of how the geometric ideas expressed in the Temperley-Lieb category have natural connections to a central topic in Logic and Computation.

\section{Further Directions}

We hope to have given an indication of the rich and suggestive connections which exist between ideas stemming from knot theory, topology and mathematical physics, on the one hand, and logic and computation on the other, with the Temperley-Lieb category serving as an intuitive and compelling meeting point. We hope that further investigation will uncover deeper links and interplays, leading to new insights in both directions.

We conclude with a few specific directions for future work: 
- The symmetric case, where we drop the planarity constraint, is also interesting. The algebraic object corresponding to the TemperleyLieb algebra in this case is the Brauer algebra [12], important in the representation theory of the Orthogonal group (Schur-Weyl duality). Indeed, there are now a family of various kinds of diagram algebras: partition algebras, rook algebras etc., arising in quantum statistical mechanics, and studied in Representation Theory [25].

- The categorical perspective suggests oriented versions of the TemperleyLieb algebra and related structures, where we no longer have $A=$ $A^{*}$. This is also natural from the point of view of Quantum Mechanics, where this non-trivial duality on objects distinguishes complex from real Hilbert spaces.

- We can ask how expressive planar Geometry of Interaction is; and what rôle may be played by braiding or other geometric information.

- Again, it would be interesting to understand the scope and limits of planar Quantum Mechanics and Quantum information processing.

\section{References}

[1] S. Abramsky. Retracing some paths in process algebra. In U. Montanari and V. Sassone, editors, Proceedings of CONCUR '96, volume 1119 of Springer Lecture Notes in Computer Science, pages 1-17. Springer-Verlag, 1996.

[2] S. Abramsky. High-level methods for quantum computation and information. In Proceedings of the 19th Annual IEEE Symposium on Logic in Computer Science, pages 410-414. IEEE Computer Science Press, 2004.

[3] S. Abramsky. Abstract scalars, loops, and free traced and strongly compact closed categories. In J. Fiadeiro, editor, Proceedings of CALCO 2005, volume 3629 of Springer Lecture Notes in Computer Science, pages 1-31. Springer-Verlag, 2005. 
[4] S. Abramsky and B. Coecke. A categorical semantics of quantum protocols. In Proceedings of the 19th Annual IEEE Symposium on Logic in Computer Science, pages 415-425. IEEE Computer Science Press. quant-ph/0402130, 2004.

[5] S. Abramsky and B. Coecke. Abstract physical traces. Theory and Applications of Categories, 14:111-124, 2005.

[6] S. Abramsky and R. W. Duncan. A categorical quantum logic. Mathematical Structures in Computer Science, 16:469-489, 2006.

[7] S. Abramsky, E. Haghverdi, and P. J. Scott. Geometry of interaction and linear combinatory algebras. Mathematical Structures in Computer Science, 12:625-665, 2002.

[8] S. Abramsky and R. Jagadeesan. New foundations for the geometry of interaction. Information and Computation, 111:53-119, 1994.

[9] H. P. Barendregt. The Lambda Calculus, volume 103 of Studies in Logic. North-Holland, 1984.

[10] C. H. Bennet, G. Brassard, C. Crépeau, R. Jozsa, A. Peres, and W. K. Wooters. Teleporting an unknown quantum state via dual classical and Einstein-Podolsky-Rosen channels. Physical Review Letters, 70:1895-1899, 1993.

[11] G. Birkhoff and J. von Neumann. The logic of quantum mechanics. Annals of Mathematics, 37:823-843, 1936.

[12] R. Brauer. On algebras which are connected with the semisimple continuous groups. Ann. Math., 38:854-872, 1937.

[13] W. Buszkowski. Mathematical linguistics and proof theory. In J. van Benthem and A. ter Meulen, editors, Handbook of Logic and Language, chapter 12, pages 683-736. Elsevier, 1997.

[14] A. Church. The Calculi of Lambda Conversion. Princeton University Press, 1941.

[15] B. Coecke. De-linearizing linearity: projective quantum axiomatics from strong compact closure. Electronic Notes in Theoretical Computer Science, 2006. To appear.

[16] B. Coecke and E. O. Paquette. Generalized measurements and Naimark's theorem without sums. To appear in Fourth Workshop on Quantum Programming Languages, 2006. 
[17] B. Coecke and D. Pavlovic. Quantum measurements without sums. In G. Chen, L. Kauffman, and S. Lamonaco, editors, Mathematics of Quantum Computing and Technology. Taylor and Francis, 2006. To appear.

[18] J. Diestel. Graph Theory. Springer-Verlag, 1997.

[19] P. A. M. Dirac. The Principles of Quantum Mechanics (third edition). Oxford University Press, 1947.

[20] K. Dosen and Z. Petric. Self-adjunctions and matrices. Journal of Pure and Applied Algebra, 184:7-39, 2003.

[21] P. Freyd and D. Yetter. Braided compact closed categories with applications to low-dimensional topology. Advances in Mathematics, 77:156-182, 1989.

[22] J.-Y. Girard. Linear Logic. Theoretical Computer Science, 50(1):1$102,1987$.

[23] J.-Y. Girard. Geometry of Interaction I: Interpretation of System F. In R. Ferro, editor, Logic Colloquium '88, pages 221-260. NorthHolland, 1989.

[24] D. Gottesman and I. L. Chuang. Quantum teleportation is a universal computational primitive. Nature, 402:390-393, 1999.

[25] T. Halvorson and A. Ram. Partition algebras. European J. of Combinatorics, 26(1):869-921, 2005.

[26] V. F. R. Jones. A polynomial invariant for links via von Neumann algebras. Bulletin of the Amer. Math. Soc., 129:103-112, 1985.

[27] A. Joyal, R. Street, and D. Verity. Traced monoidal categories. Mathematical Proceedings of the Cambridge Philosophical Society, 119:447-468, 1996.

[28] C. Kassel. Quantum Groups. Springer, 1995.

[29] L. H. Kauffman. An invariant of regular isotopy. Trans. Amer. Math. Soc., 318(2):417-471, 1990.

[30] L. H. Kauffman. Knots in Physics. World Scientific Press, 1994.

[31] L. H. Kauffman. Knot Logic. In L. H. Kauffman, editor, Knots and Applications, pages 1-110. World Scientific Press, 1995. 
[32] L. H. Kauffman. Biologic II. In N. Tongring and R. C. Penner, editors, Woods Hole Mathematics, pages 94-132. World Scientific Press, 2004.

[33] L. H. Kauffman. Knot diagrammatics. In W. W. Menasco and M. Thistlethwaite, editors, Handbook of Knot Theory. Elsevier, 2005.

[34] G. M. Kelly and M. L. Laplaza. Coherence for compact closed categories. Journal of Pure and Applied Algebra, 19:193-213, 1980.

[35] M. Laforest, R. Laflamme, and J. Baugh. Time-reversal formalism applied to maximal bipartite entanglement: Theoretical and experimental exploration. quant-ph/0510048. Unpublished.

[36] J. Lambek. The mathematics of sentence structure. Amer. Math. Monthly, 65:154-170, 1958.

[37] R. Milner. Fully abstract models of typed lambda-calculus. Theoretical Computer Science, 4:1-22, 1977.

[38] M. Moortgat. Categorial type logic. In J. van Benthem and A. ter Meulen, editors, Handbook of Logic and Language, chapter 2, pages 93-177. Elsevier, 1997.

[39] R. Raussendorf and H.-J. Briegel. A one-way quantum computer. Physical Review Letters, 86:5188, 2001.

[40] R. Raussendorf, D. Browne, and H.-J. Briegel. Measurementbased quantum computation on cluster states. Physical Review A, 68:022312, 2003.

[41] M. Rédei. Why John von Neumann did not like the Hilbert space formalism of quantum mechanics (and what he liked instead). Studies in History and Philosophy of Modern Physics, 27:493-510, 1997.

[42] P. Selinger. Dagger compact closed categories and completely positive maps. Electronic Notes in Theoretical Computer Science, 2006. To appear.

[43] H. N. V. Temperley and E. H. Lieb. Relations between the 'percolation' and 'coloring' problem and other graph-theoretical problems associated with regular planar lattices: some exact results for the 'percolation' problem. Proc. Roy. Soc. Lond. A, 322:251-280, 1971. 
[44] E. Witten. Topological quantum field theory. Communications in Mathematical Physics, 117:353-386, 1988.

[45] M. Zukowski, A. Zeilinger, M. A. Horne, and A. K. Ekert. 'Event-ready-detectors' Bell experiment via entanglement swapping. Physical Review Letters, 71:4287-4290, 1993.

CRC PRESS

Boca Raton Ann Arbor London Tokyo 